\title{
Shape Optimization of Wind Turbine Airfoils by Genetic Algorithm
}

\author{
A. R. Davari, M. Hasheminejad, and A. Boorboor
}

\begin{abstract}
Wind Turbine airfoil was optimized by a new genetic method so that its performance increases. A new method was proposed to optimize thin airfoils for use in wind turbine rotors. It is a common practice in the classic methods based on the genetic algorithms for airfoil shape optimizations, to use the computational fluid dynamics solvers. The main disadvantage of this method is the considerable running time for these algorithms. This disadvantage was significantly reduced by using the new technique. Comparing the results of the proposed algorithm with the classic ones approves its higher performance and lower computation time. Another advantage of this method is the reduced number of the design variables needed for optimization by inverse design method. The proposed modifications on the genetic algorithm offer a higher performance wind turbine blades faster than classic algorithms
\end{abstract}

Index Terms-Intelligent genetic algorithm, thin airfoil theory, airfoil optimization, inverse design method.

\section{INTRODUCTION}

Competition between all types of industries about cost, performance and time brought the optimization problems into fore front. Optimization is a useful tool for improving methods for higher performance and lower running time. The modern optimization methods As artificial neural network, the evolutionary algorithms and the genetic algorithm, which has been introduced in this paper, are most usual and popular than the older approaches because of their more flexibilities and higher performance for optimizing all kind of aerodynamic problems.

Airfoil performance is an important parameter in the efficiency of the wind turbines. The airfoil maximum lift, its maximum lift to drag ratio and its post stall behavior all affect the overall performance of the wind turbine. In addition, the pitching moment developed by the airfoil also has a strong effect on the behavior and performance of the wind turbines. Therefore, a good airfoil selection and design still remais to be an important problem. There are two common approaches to optimize the wind turbine blade sections. The first, the direct method consists of selecting a known airfoil shape with performance similar to that required by the new application and making slight modifications to the shape to reach the required performance. In the second approach, known as the inverse method, the designer specifies the performance characteristics required by the airfoil and uses computer programs to compute the airfoil geometry that produces the desired performance. The advantage of direct approach is its

Manuscript received September 19, 2012; November 27, 2012.

The authors are with Department of Mechanical and Aerospace Engineering, Science and Research Branch, Islamic Azad University, Tehran, Iran (e-mail: ardavari@sr.iau.ac.ir, sm.hasheminejad@gmail.com, Arash@Boorboor.com). simplicity. However, the classical direct approaches mostly perform a local search. The inverse approach The inverse approach is far more powerful since the designer has much more precise control over the final performance of the airfoil. However, while every airfoil shape produces a particular set of performance characteristics, not every set of performance characteristics can be used to generate a realistic airfoil shape. The designer must be aware of what is practical, the trade-offs required between different types of performance, and physical constraints. As a tradeoffs required between the different types of performance, and physical constraints. Consequently, classical design and optimizing methods mainly adopt "trial and error" approach and strongly rely on designer's future experience rather than the current needs. A global optimization method based on genetic algorithm or intelligent genetic algorithm is expected to shorten and simplify the iterative design process and improve the design output. A similar approach has been previously used for aerodynamic optimization of turbo machinery cascades [1], and very good results were obtained. Glaurt [2] neglected the drag of airfoil and offered a popular theory for optimizing the airfoil. This theory was further developed by Stewart [3] and improved by Miller [4]. Wilson [5] offered a method base on calculating sensitivity of flow field parameters.

\section{METHOD}

The wind turbine shape is expressed analytically as a function of some design parameters. The design parameters of the NACA 4 digit airfoils are used to control the camber shape and thickness of the wind turbine blade sections.

The solver has to provide fast and robust computation of the lift, pitching moment and drag of the airfoil of wind turbine placed in a low-speed viscous flow. A one-way coupled inviscid - boundary layer model is used for solving numerically the thin wind turbine equation. The lift coefficient of the blade section is obtained from Eq. (1). This equation is used as the fitness function of the genetic algorithm

$$
\begin{gathered}
C_{l}=C_{l_{0}}+\frac{d C_{l}}{d \alpha} \alpha=\pi\left(A_{1}-A_{0}\right)+2 \pi \alpha \\
C_{m_{1 / 4}}=\pi / 4\left(A_{1}-A_{0}\right)
\end{gathered}
$$

where $A_{0}$ and $A_{1}$ are defined in reference [6]. The moment coefficient in Eq. (2) is calculated about the quarter chord. Airfoil shape can be determined using Eq. (3) and (4), where Eq. (3) is for thickness and Eq. (4) is for the mean camber line shape of the thin airfoil. And camber line derivations are given by Eq. (5) [7]. the genetic algorithm use Eq. (4) and (5) 
for creating the new camber line in optimization process that the optimized camber will be have better aerodynamic behavior.

$$
\begin{gathered}
\frac{y_{t}}{c}= \pm \frac{t}{c}\left[B_{0} \sqrt{\xi}-\mathrm{B}_{1} \xi-B_{2} \xi^{2}-B_{3} \xi^{3}\right] \\
y_{c}=(m c / p)\left[2 p \xi-\xi^{2}\right] \quad \xi \leq P \\
y_{c}=\frac{m c}{(1-P)^{2}}\left[(1-2 P)+2 P \xi-\xi^{2}\right] \quad \xi \geq P \\
\frac{d y_{c}}{d x}=\frac{2 m c}{(1-p)^{2}}(p-\xi) \quad \xi \geq P \\
\frac{d y_{c}}{d x}=\frac{2 m c}{p^{2}}(p-\xi) \quad \xi \leq P
\end{gathered}
$$

where $z=x / c$ and the value of coefficients are $B 0=1.4845$, $B 1=0.6300, B 2=1.7580, B 3=1.4215$ and $B 4=0.5075$. And the solution strategy in the proposed algorithm is to generate all of the possible random members of the first generation from the initially given airfoil. Using elite selection, random selection, mutation and cross over methods in the genetic algorithms [8], produce the next generation. This loop continues until the design criterion is met. At this stage, the obtained airfoil shape would be the optimum one.

\section{RESULTS}

Thin airfoils such as NACA 4412 are mostly used in wind turbine blade section [9]. This particular airfoil is used to demonstrate the performance of the present method in comparison with the classic approach. However, both methods can be used for any four digits NACA airfoils. The optimum airfoil obtained by the present method has been compared with the NACA 4412 as the initially given airfoil in Fig. 1. The difference is obviously in the value and the location of the maximum camber.

In Fig. 2, the variation of the camber to chord ratio of each member in the second generation is compared for both methods. As can be observed, the present method has less fluctuation and exhibits an ascending behavior, while in the classic method, a high amplitude fluctuation is observed in the camber to chord ratio of the members. The smooth behavior of the aforementioned parameter in the present method assures a fast convergence to the optimum result with a higher efficiency. Note that the last member, i.e. the 100th member, in both methods, nearly reaches to the same value. However, the in present method, this final value is achieved with less variation in the amplitude, while in the classic approach, a chaotic variation in the amplitude is observed.

Convergence and Mean fitness function behavior is depended on the genetic factor values so that the code is converging only with correct magnitude of all of them in other hand each factors has its role on convergence. Fig. 3 shows the effect of each genetic factor without concerning other factors. Some factors are stabilizer and others are dynamic.
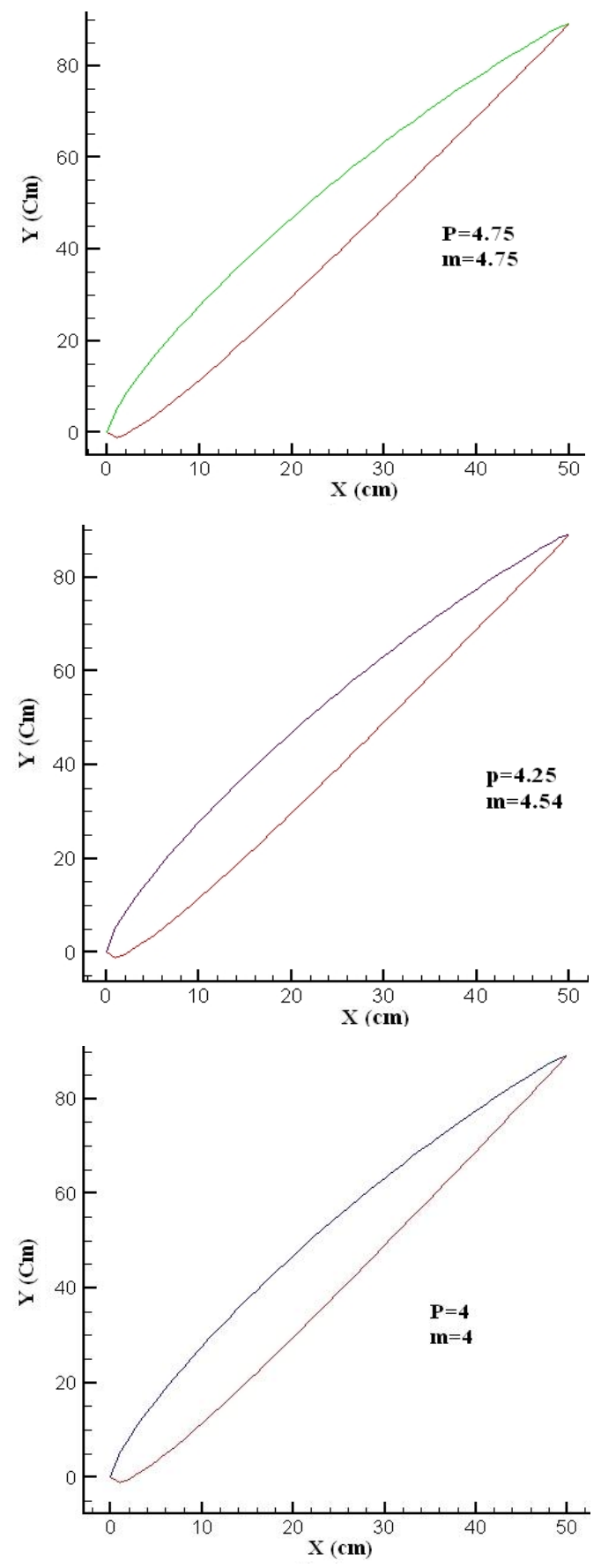

Fig. 1. Primary and optimum airfoils.

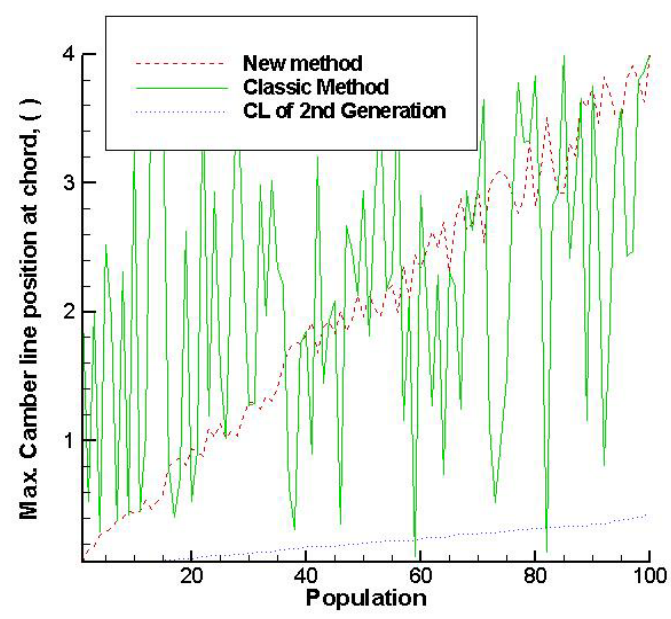

Fig. 2. The camber to chord ratio for each member in the second generation 


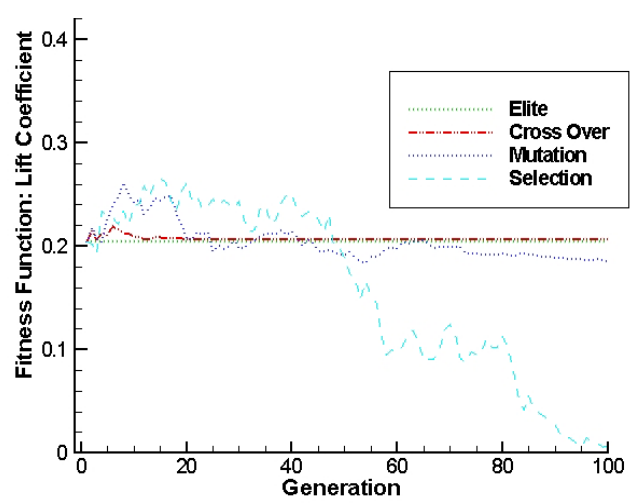

Fig. 3. The effects of each genetic factor on mean fitness function at 100 generation period

Fig. 4 shows some curves that they were drawn for some generation by gathering the value of fitness function for each population so that the convergence can be define by horizontal line which means that all of the population reach to the certain amount of fitness or lift coefficient. In Fig. 4 the curves were turned to horizontal by increasing the generation

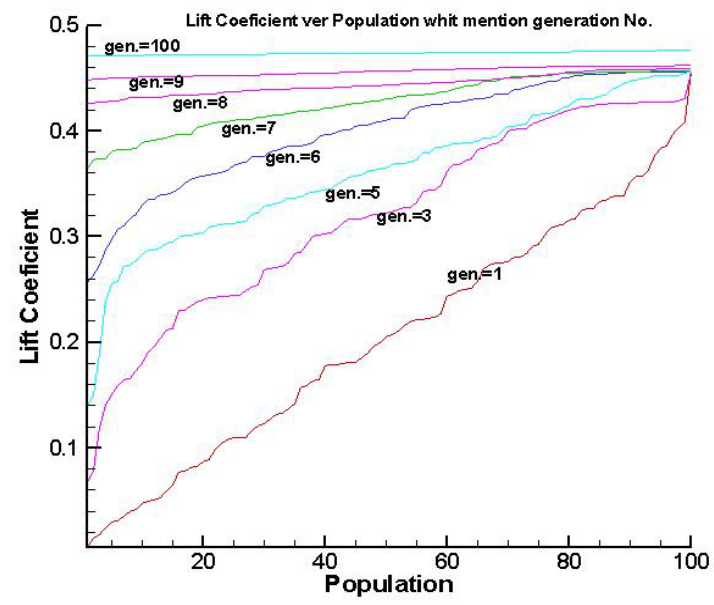

Fig. 4. The fitness values (Lift coefficient) versus population for some generation.

The population is more important than generation in convergence. Fig. 5 shows the convergence surface that generation is laid on $\mathrm{x}$-axis and $\mathrm{Y}$ direction shows the population. The optimized airfoil is obtained at 18 generation. The horizontal line is described the convergence so that the algorithm is convergence at the first horizontal line as shown by Fig. 5

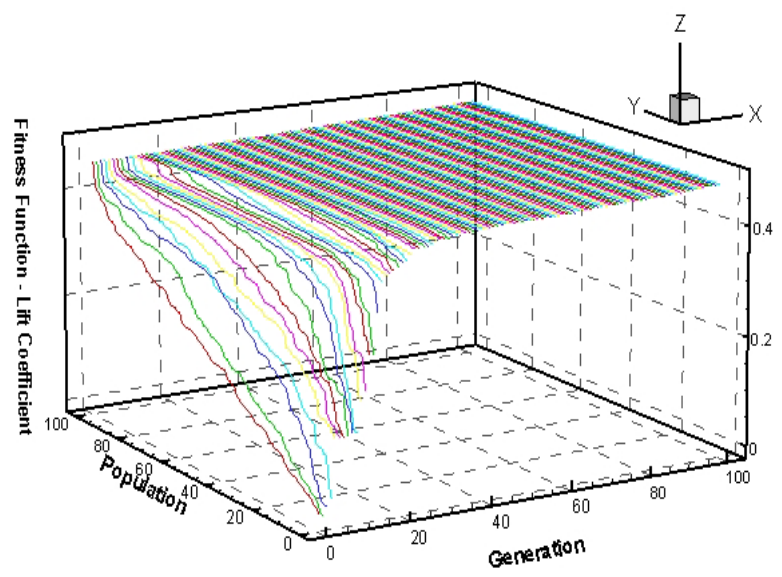

Fig. 5. The surface of fitness function-population-generation
Fig. 6 shows the effect of cross-over probability on the convergence trajectory so that smoother curves are belonged to the higher probability. In Fig. 6 the curve of probability for 0.1 has sharp local slop and too many fluctuations. And curve for higher probability are smoother

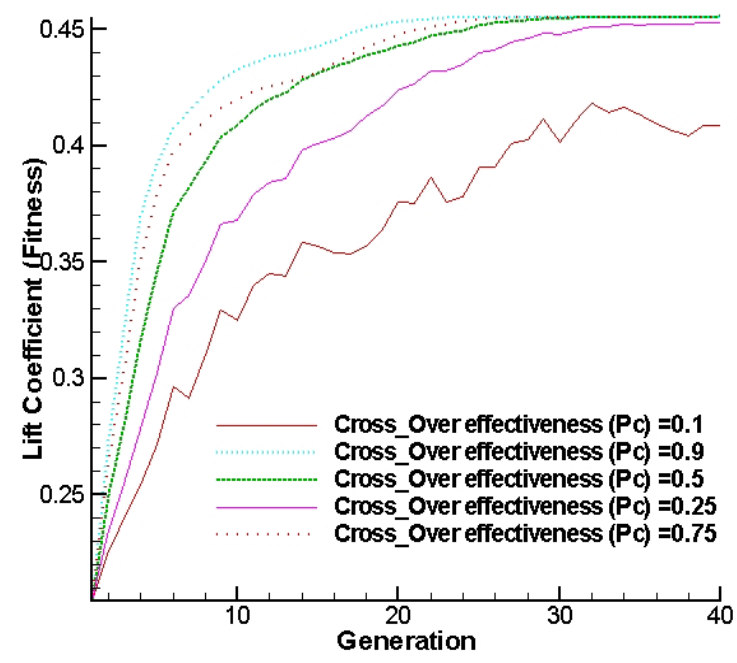

Fig. 6. Cross-over probability

\section{CONCLUSION}

A method was proposed to be used in the genetic algorithm to optimize the NACA four digit series thin airfoils of the wind turbine rotors. The optimum airfoils obtained by this method were compared with those obtained by the classic methods.

The results show a remarkable improvement in the performance of the genetic algorithm in airfoil optimization problems. The higher aerodynamic performance of the optimized airfoil along with lower computational effort is the most important features of the present method in comparison to the classic ones.

And also the results show that when new method just was used only one of the genetic factors, any of the them have no positive effects for algorithm converging however the mixed of all genetic factors that coefficients of genetics factors rate sets precisely for it caused a fast and high performance converging in new method genetic algorithm to the final optimum airfoil or wind turbine section.

The curves of converging that shows the fitness function (Lift coefficient) versus generation are similar to exponential function although they are raised in the first and then their slops decrease slowly until these curve turn to constant value from a one specific generation number. So this behavior shows that the mutations and fluctuations are enormous intensively at the several begging generations and then curves are find the smoother behavior due to damping the begging vibrations and fluctuations then the curves converges to the certain fitness value

This study shows that elite and cross over factors are neutral in genetic algorithms however they have stability effects on the entire trajectory of converging. Cross over neutralize behavior in new method is against of its behavior at the classic method because cross over factor has changed in new genetic algorithm method. And also mutation and random selection factors are unstable but they were made a 
local fluctuation. And the new genetic algorithm method are used these local fluctuation for improve the total fitness value of the next generation

Mutation factor effects as vibration and fluctuation factor on genetic algorithm so that its factor can be caused to converging or even diverging the responses in other hand mutation makes the local maximums and minimums and then these maximums have directed to global maximum by using the précised amount of other factors as it seen mutation has bad results in Fig. 3 when genetic algorithm use it alone but its good features are expected when it used together with other genetic factors

The slop of curves are increasing by decreasing the cross-over probability from parents with lower fitness due to increasing the new point and cause the wider searching however the faster and smoother convergence is gotten by increasing the cross-over probability

\section{REFERENCES}

[1] O. Oksuz, I. S. Akmandor, and M. S. Kavsaoglu, "Aerodynamic Optimization of Turbomachinery Cascades Using Euler/Boundary Layer Coupled Genetic Algorithms," Journal of Propulsion and Power, vol. 18, no. 3, pp. 652-657, May 2002.

[2] D. A. Spra, Wind Turbine Technology, New York, ASME press, 1994.

[3] H. J. Stewart, "Dual Optimum Aerodynamic Design for Horizontal Axis Wind Turbines," AIAA Journal, 1976.

[4] D. R. Miller, Wind Turbine Strutural Dynamics, NASA Conference Publication, 1977.

[5] D. M. E. Forrest, Wind Turbine Engineering Design, New York, Vannostrad Rebhold Company, 1987.

[6] E. L. Houghton and P. W. Carpenter, Aerodynamics for Engineering Student. $5^{\text {th }}$ ed., Butterworth-Heinemann, London, pp. 189-196, 2003.

[7] T. Burton, D. Sharpe, N. Jenkins, and E Bossanyi, Wind Energy Handbook, John Wily and Sons Ltd., Chichester, 2001.
[8] L. H. Hartwell, L. Hood, and L. Mikhael, Goldberge Genetics from Gene to Genomes, McGraw Hill, pp. 24-38, 2001.

[9] J. D. Anderson, Fundamentals of Aerodynamics, McGraw-Hill, 2001.

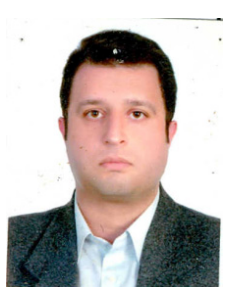

Ali R. Davari was born in 1976 and is assistant professor in the department of mechanical and aerospace engineering at the science and research campus of the Islamic Azad University in Iran. He received his $\mathrm{PhD}$ degree in Aerospace Engineering at Sharif University of Technology, Iran in 2006. He has conducted several experimental studies on unsteady aerodynamics and interference effects. His current research interests are new prediction and optimization methods in aerodynamics, such as neural networks and evolutionary algorithms. Dr. Davari is a member of IAS and ISME in Iran and has already presented over 50 papers in international conferences and published over 12 journal papers.

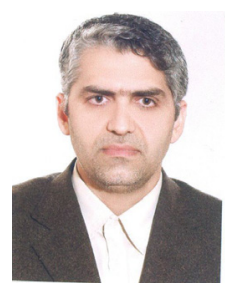

Mahmoud Hashemi Nejad was born in Iran $\mathrm{He}$ received his B.S. in Electrical Engr. from Chamran University, Ahwaz, Iran, in 1988. M.S. and $\mathrm{PhD}$ in Control Engr. in 1993 and 1996 respectively from Kyushu university, Fukuoka, Japan. He is currently an assistant Professor of Department of Energy and Environment in Material \& Energy Research Institute, Karaj, Iran. His research interest are control system design, renewable energies and automation.

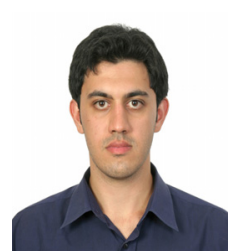

Arash Boorboor was born in Iran on 12 December 1984. He received his B.S. and M.S. in Aerospace Engr. in 2009 and 2012 respectively from Science and Research Campus of the Islamic Azad University, Tehran, Iran. He was worked on some project in Iran Space Agency and has already presented 3 papers in international conferences 